\title{
Pulmonary necrobiotic nodules as a presenting feature of rheumatoid arthritis
}

\author{
SALLY HULL AND JOHN A. MATHEWS \\ From the Department of Rheumatology, St Thomas's Hospital, London
}

SUMMARY In rheumatoid disease only 4 cases have been reported in which lung nodules preceded arthritis. Two further cases are presented in which intrapulmonary nodules were noted at the onset of the disease, thus provoking a diagnostic dilemma. In the first patient rheumatoid factor tests were initially negative, and diagnostic thoracotomy was performed. In the second patient serological tests were positive, and biopsy was avoided. It therefore seems that detection of circulating rheumatoid factor in patients with lung shadows can be a useful aid to diagnosis and management.

Arthritis is far the commonest feature of rheumatoid disease. However, systemic aspects are not infrequent and the respiratory system may be involved in several ways. These include pleurisy, necrobiotic nodules, Caplan's syndrome (rheumatoid pneumoconiosis), diffuse interstitial pulmonary fibrosis (fibrosing alveololitis), and pulmonary artery involvement.

Pulmonary necrobiotic rheumatoid nodules are a rare manifestation of the disease, but when they precede arthritis or the detection of circulating rheumatoid factor they can present an important diagnostic problem. Four such cases have previously been reported, ${ }^{12}$ and 2 more are described here.

We present 2 patients in whom lung nodules were noted concurrently with the first symptoms of rheumatoid arthritis. Both cases are notable for the virtually simultaneous detection of pulmonary shadows. In one of the patients the serological tests for rheumatoid disease became positive in time for him to escape lung biopsy. We suggest that a personal or family history of rheumatoid disease and serological tests for rheumatoid arthritis are worth including early in the search for the aetiology of discrete lung shadows.

CASE 1

A 46-year-old male finance officer presented in October 1978 with a 6-weeks history of pain in the right shoulder, which first appeared after exercise. A few weeks later this developed into a polyarthralgia affecting both shoulders, wrists, and

Accepted for publication 20 January 1981.

Correspondence to Dr S. Hull, Department of Rheumatology, The London Hospital, Whitechapel, London E1 1BB. proximal interphalangeal joints. His past medical history included a Ghon focus in the right lung, noted in 1967, and in 1976 a transient synovitis of the right knee which developed after dancing. He smoked 10 cigarettes a day, and has a brother with rheumatoid arthritis.

Physical examination showed an olecranon bursitis, though there was no active synovitis of peripheral joints and subcutaneous nodules were not detected. The chest $x$-ray (Fig. 1a) showed numerous coin-like opacities throughout the lung fields. These ranged in size from $0.5 \mathrm{~cm}$ to $2.3 \mathrm{~cm}$ and some showed cavitation, seen most clearly on tomography (Figs. 1b, 1c). There was also one focus of calcification which coincided with the previously noted Ghon focus. The erythrocyte sedimentation rate was $47 \mathrm{~mm}$ in the first hour, Rose-Waaler titre less than 1 in 4, latex test negative (in blood and in fluid aspirated from the olecranon bursa), and the immunofluorescent antinuclear factor insignificant at 1 in 10. Sputum culture was negative for acidfast bacillus. Complement fixation for candida species and the Aspergillus fumigatus precipitin test were negative. Lung function tests showed a restrictive defect with a reduced transfer factor, $15.9 \mathrm{ml} \mathrm{CO} / \mathrm{min} / \mathrm{mmHg}$ ( $75 \%$ of predicted value). Owing to the large number of opacities, the negative serology, and their sinister appearance a histological diagnosis was sought. Needle biopsy and then bronchoscopy were performed in an attempt to reach a histological diagnosis, but on both occasions necrotic or normal material was obtained. But open thoracotomy yielded tissue whose histology had the characteristics of a rheumatoid lung nodule (Fig. 2). Immediately after the operation his arthralgia 


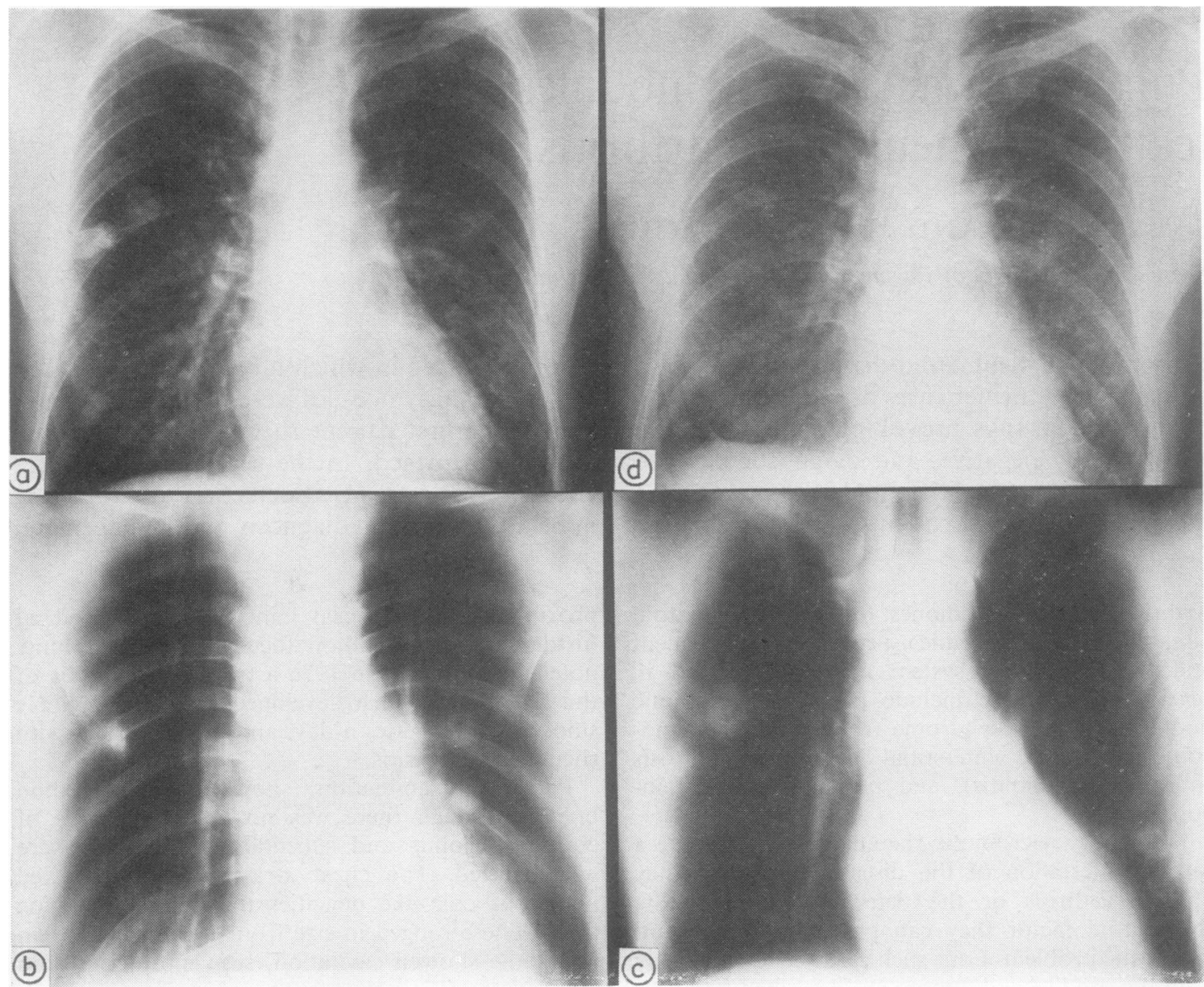

Fig. 1 (a) Numerous coin-like opacities at presentation. (b) Tomographs clearly demonstrate cavitation and the (c) pre-existing Ghon focus. (d) The opacities regressed over 16 months.

disappeared and the Rose-Waaler titre remained negative, but 6 months after his initial presentation he had a recurrence of joint pains, later accompanied by mild swelling with morning stiffness. The RoseWaaler titre rose to 1 in 2048. His arthritis remains symptomatically controlled on nonsteroidal antiinflammatory drugs. By February 1980, 18 months after presentation, the rheumatoid factor titre had fallen to 1 in 4 , the erythrocyte sedimentation rate (ESR) to $25 \mathrm{~mm}$, and there was considerable reduction in the number of radiologically apparent lung nodules (Fig. 1d). Hand $x$-rays remained nonerosive.

CASE 2

A 54-year-old male clerk was seen in the Chest Department of St Thomas's Hospital in March 1966 with an opacity in the upper zone of his right lung (Fig. 3a). This had been noted at mass miniature radiography which he had attended because of persistent 'flu-like' symptoms. Three months later he developed a polyarthritis affecting wrists, elbows, and proximal interphalangeal joints. The RoseWaaler titre was 1 in 2048, antinuclear factor less than 1 in 4, and sputum was negative for acid-fast bacilli. Chest $x$-ray (Fig. 3b) now showed cavitation of the initial lesion and the appearance of a second opacity in the left mid-zone. Lung function tests showed a slight restrictive defect with a low transfer factor of $9.1 \mathrm{ml} \mathrm{CO} / \mathrm{min} / \mathrm{mmHg}(50 \%$ of predicted value). Lung biopsy was not performed, as it was now assumed that the lung lesions were related to his rheumatoid disease. He was admitted for control of 


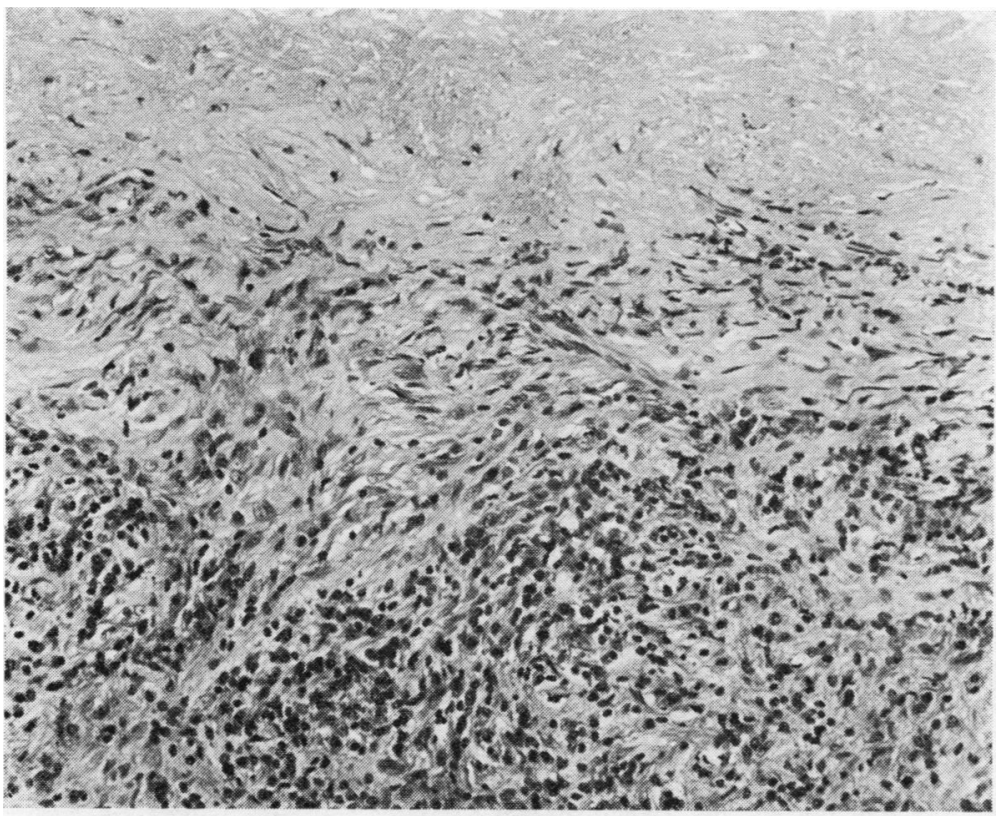

Fig. 2 Section of necrobiotic lung nodule removed at thoracotomy.

active arthritis and discharged on phenylbutazone, having developed a rash while on gold injections.

In March 1967 subcutaneous nodules and microinfarcts on the fingers were noted. By this time the chest $x$-ray showed cavitation of both opacities (see Fig. 3c) but no fresh lesions. In June 1967 he had a transient cerebral ischaemic attack and was found to be hypertensive. Investigation of renal function showed a creatinine clearance of $37 \mathrm{ml} / \mathrm{min}$, and a renogram showed bilateral poorly functioning kidneys. By this time the lung fields were clear of nodules (Fig. 3d). He died soon after a second cerebrovascular accident in 1970 , but no postmortem was performed.

\section{Discussion}

Necrobiotic pulmonary nodules are well established among the extra-articular manifestations of rheumatoid arthritis. ${ }^{13}$ The nodules may be single or multiple, ranging in size from a few millimetres to $7 \mathrm{~cm}$ or more. In a series of 516 patients studied 2 cases with lung nodules were found ${ }^{4}$ The nodules may multiply or regress independently of the disease process elsewhere, and during their existence they may calcify ${ }^{5}$ or cavitate ${ }^{6-8}$ and even lead to pyopneumothorax. ${ }^{9}$ Associated symptoms include cough or haemoptysis. Surprisingly, necrobiotic nodules occur more frequently in men than women, with a predominance of $7: 3$, compared to the $2 \cdot 5: 1$ female predominance of rheumatoid arthritis. ${ }^{7}$

The majority of recorded intrapulmonary nodules have been in patients with longstanding seropositive rheumatoid disease. However, Scadding reports 1 case $^{1}$ and Eraut et al. ${ }^{2} 3$ cases in which necrobiotic pulmonary nodules preceded the appearance of seropositive rheumatoid disease. The latent period ranged from 6 months to 11 years, and during this time serological tests for rheumatoid disease were not recorded.

Both our patients presented with discrete pulmonary lung shadows in need of a diagnosis. In the second case the rapid emergence of seropositive nodular rheumatoid arthritis was deemed adequate confirmation of the diagnosis and the lung lesion was not biopsied. In the first case an early histological diagnosis was thought to be mandatory, as rheumatoid factor tests were negative. It is salutary that needle biopsy and bronchoscopy failed to provide adequate tissue, yielding only necrotic material from the centres of cavitation, and diagnosis was confirmed only after open thoracotomy.

It is clear both from our cases and those described by Eraut et al. ${ }^{2}$ that necrobiotic lung nodules, either singly or in large numbers, may be a cause of lung opacity even before the appearance of rheumatoid arthritis. A search for rheumatoid factor should be included in the early diagnostic tests. 


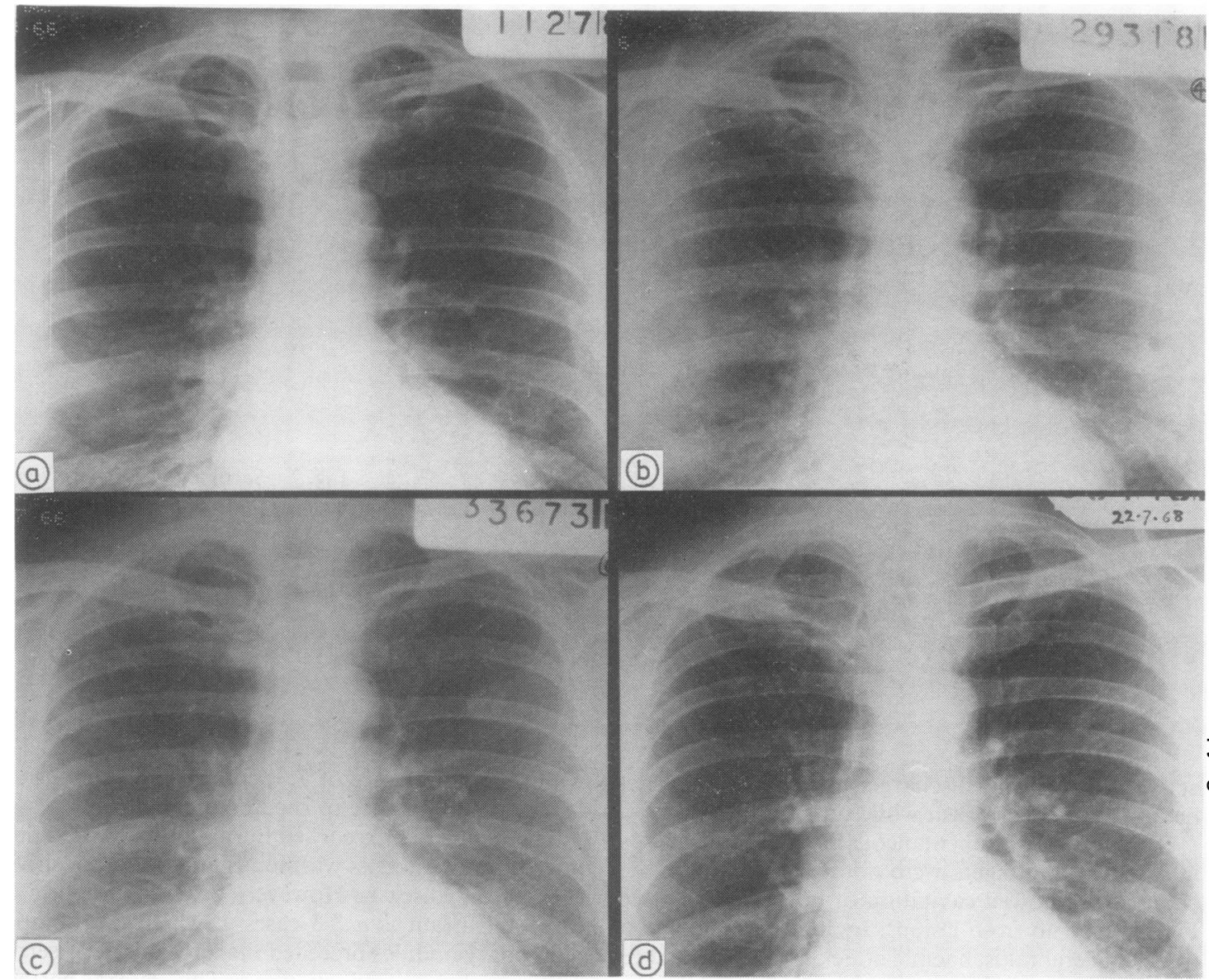

Fig. 3 (a) A single opacity in the right upper zone on presentation. (b) This opacity cavitates and a left upper zone opacity is seen. (c) The left upper zone cavity also cavitates. (d) All lesions regress over a 3-year period.

\section{References}

1 Scadding J G. The lungs in rheumatoid arthritis. Proc $R$ Soc Med 1969; 62: 227-38.

2 Eraut C D, Evans J A, Caplin M. Pulmonary necrobiotic nodules without rheumatoid arthritis. $\mathrm{Br} \mathrm{J}$ Dis Chest 1978; 72: 301-6.

3 Ellman P, Cudkowicz L, Elwood J S. Widespread serous membrane involvement by rheumatoid nodules. $J$ Clin Path 1954; 7: 239-44.

4 Walker W C, Wright V. Pulmonary lesions and rheumatoid arthritis. Medicine (Baltimore) 1968; 47: 501-20.
5 Mattingly S. The lungs and rheumatoid arthritis. Ann Phys Med 1964; 7: 185-202.

6 Noonan C D, Taylor F B, Engelman E P. Nodular rheumatoid disease of the lung with cavitation. Arthritis Rheum 1963; 6: 232-40.

7 Yates D A H. Cavitation of a rheumatoid lung nodule. Ann Phys Med 1963; 7: 105-6.

${ }^{8}$ Panettiere F, Chandler B F, Libeke J R. Pulmonary cavitation in rheumatoid disease. Am Rev Resp Dis 1968; 97: 89-95.

9 Hindle W, Yates D A H. Pyopneumothorax complicating rheumatoid lung disease. Ann Rheum Dis 1965; 24: 57-60. 